\title{
Suppressing Breakups in Binary Droplet Collisions by Surfactant
}

\author{
Kuan-Ling Huang*, Kuo-Long Pan, Jen-Chun Chen \\ Department of Mechanical Engineering, National Taiwan University, Taiwan \\ ${ }^{*}$ Corresponding author email : sliderhuang@gmail.com
}

\begin{abstract}
In collisions of two droplets, reflexive separation (IV), rotational separation (VI) and stretching separation (V) usually occur after temporary coalescence at sufficiently large Weber number $(W e)$ within various ranges of impact parameter $(B)$ and Ohnesorge number $(O h)$. Here we present the influence of surfactant on various separation regimes by experiments. Compared to the surfactant-free cases in head-on collisions, adding surfactant delays the occurrence of regime (IV), showing larger values of the critical We for transition (denoted by $W e_{c}$ ) between the regimes of coalescence (III) and reflexive separation (IV). By increasing surfactant concentration over the critical micelle concentration (CMC), We $e_{c}$ decreases and approaches the theoretical value predicted by energy conservation. Regarding the off-center droplet collisions without adding surfactant, we have rederived the theoretical model proposed by Jiang et al. to predict the transition boundary between regimes (III) and (V). The results show that our model, in a dimensionless form, describes the existing experimental data with a higher precision compared to the previous model having dimensional fitting coefficients and it is valid for surfactant-free drops in a range of $O h=0.0089-0.0592$. When surfactant is added, however, about $30 \%$ larger $B$ is required to reach regime (V). Our experiments exclusively demonstrate the influence of surfactant on suppressing the separations in binary droplet collisions. The cause can be interpreted by the non-uniform distributions of surfactant on the droplet surfaces, which induce Marongoni stresses that oppose droplet deformation and further breakup.
\end{abstract}

\section{Keywords}

Droplet collision, Surfactant, Stretching separation, Reflexive separation, Rotational separation.

\section{Introduction}

Droplet collisions in a gaseous medium are ubiquitous in various natural and practical processes, such as raindrop formation [1,2], spray combustion in liquid-fueled engines $[3,4]$, and the operation of nuclear reactors $[5,6]$. In the past decades, various collision outcomes of two identical drops were unveiled by delicate experiments [7-12] and mapped in a regime diagram depicted by the Weber number $\left(W e=\rho_{l} U D / \sigma\right)$ and an impact parameter $(B=x / D)$. As shown in Fig. 1(a), these collision outcomes are identified as coalescence after minor droplet deformation (I), bouncing (II), coalescence after substantial droplet deformation (III), reflexive separation (IV), stretching separation (V) and rotational separation (VI). Fig. 1(b) illustrates the definitions of the collision parameters, where $U$ is the relative velocity, $D$ the diameter of the droplet, $\chi$ the projection of the separation distance between the droplet centers in the direction normal to the vector $U, \rho_{l}$ and $\sigma$ the density and surface tension of the liquid, respectively.

When droplets collide with sufficiently large We, breakups often occur with satellite droplets production [9-14]. For nearly head-on collisions, i.e., $B<0.2$, the internal rebounding flows elongate the temporarily coalesced drop and rupture the droplet surface, producing the reflexive separation (IV). At a much larger impact angle $(B>0.45)$, centrifugal forces stretch out the merged droplet and generate the stretching separation (V). More recently, the rotational separation (VI) was identified at intermediate values of $B$ [12]. This separation 
regime is caused by the coherent motions of the reflective and rotational flows inside the tentatively merged drop.

Regarding the influence of liquid properties, physical models based on momentum theory or energy conservation were proposed to describe the transition boundaries between coalescence and separation regimes [7-9,14-20]. Previously, Jiang et al. [9] proposed a sliding plate model based on the momentum theory to predict the transition from coalescence (III) to stretching separation (V). This boundary was expressed in a dimensionless form as

$$
W e^{\frac{1}{2}}=\frac{1}{B}\left[1+k \frac{\mu}{\sigma}\left(\frac{\rho r}{\sigma}\right)^{\frac{1}{2}}\right]
$$

Where $\mu$ is the liquid viscosity. In Ref. 9, the coefficient $k$ in equation (1) was considered as a constant and Gotaas et al. [13], however, showed later that this coefficient should be a dimensional term, i.e. $\mathrm{m}^{2} / \mathrm{s}^{2}$, in order to hold (1) as a dimensionless form. They modified (1) to the form

$$
B=\frac{2.656}{W e^{1 / 2}}\left[1+0.185 \frac{\mu}{\sigma}\left(\frac{\rho d}{\sigma}\right)^{\frac{1}{2}}\right] .
$$

This model describes the transition boundary (III/V) well for relatively viscous fluids $(\mu>$ $21.8 \mathrm{mpa} \cdot \mathrm{s}$ ) in Ref. 13, but fails to predict the experimental data of other Newtonian fluid systems $[17,20]$. More recently, Finotello et al. [19] established another model to predicted the occurrence of stretching separation based on energy conservation, showing

$$
\frac{W e \Delta^{2}}{12\left(1+\Delta^{3}\right)\left(1+\Delta^{2}\right)}=\frac{21}{22}(O h \sqrt{W e})^{1 / 22}\left[\frac{W e \Delta^{2}}{12\left(1+\Delta^{3}\right)\left(1+\Delta^{2}\right)}+1\right]\left[\frac{B}{1+0.250 h \sqrt{W e}}\right]
$$

where $\Delta=\frac{D_{s}}{D_{l}}=1$ for the collision between two identical droplets, $D_{s}$ and $D_{l}$ the diameters of small and large droplets, respectively. Here $O h=\mu / \sqrt{\rho D \sigma}$, is the Ohnesorge number. Equation (3) is in a dimensionless form and shows good agreements with the numerical results in Ref. 19. However, it has not been validated by any experimental data.

More recently, the influence of surfactant on the bouncing regime (II) was studied via experiments and numerical simulations [21]. It shows that Marangoni stresses, which are induced by non-uniform distributions of surfactant molecules on the droplet surface, can promote droplet bouncing after impact [21]. Considering further connection to the other regimes, here we have rederived the theoretical model proposed by Jiang et al. [9] to describe the transition boundary between regimes (III) and (V) for surfactant-free drops and carried out a series of experiments to demonstrate the surfactant effects on the generation of separation regimes.

(a)

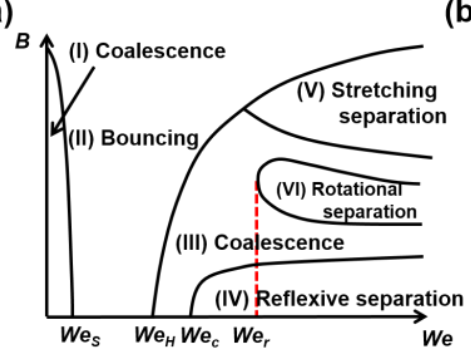

(b)

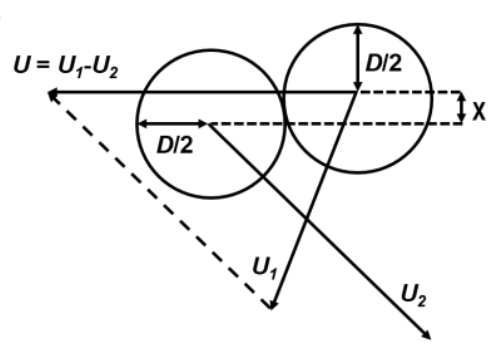

Figure 1. (a) Typical regime diagram of alkanes $[11,12,14]$. (b) Definition of parameters in binary droplet collision.

\section{Material and Methods}


The experimental setup in this work is essentially the same as that in Ref. 12. Decane, dodecane, tetradecane, hexadecane, silicone oil and water droplet with fixed diameters (300, 450 or $600 \mu \mathrm{m}$ ) were generated by the drop-on-demand (DOD) method. The droplets were squeezed out from the glass nozzles by the vibrations of piezoelectric plates, while the droplet size was primarily determined by the inner diameter of the nozzle orifices and finely adjusted by altering the pulse width of the electrical signal. On the other hand, the surfactant effect was investigated by making droplet collisions with various concentrations $(\Phi)$ of a non-ionic surfactant (S386). The properties of the working fluid are shown in Tables 1 and 2, with a wide range of $O h$ value ( 0.0044 to 0.0592 ). Moreover, droplets can be maintained spherically before collision by adjusting the distance between two nozzles. The time-resolved grayscale images were recorded by a high-speed CMOS digital camera (Vision Research, Phantom M310), which supported a maximum resolution of $512 \times 512$ pixels with 10000 frames per second (fps). The exposure time of the shutter can be set down to $1 \mu$ s to avoid image slurring. The measurement error came from the deviation between the actual droplet edge and the circle was detected by Matlab program, which was $2.1 \%$.

Table 1 - Physical properties of working fluids.

\begin{tabular}{c|cccccc}
\hline & Decane & Dodecane & Tetradecane & Hexadecane & Water & $\begin{array}{c}\text { Silicone oil } \\
(5 \mathrm{cs})\end{array}$ \\
\hline$\rho\left(\mathrm{kg} / \mathrm{m}^{3}\right)$ & 730 & 744 & 759 & 770 & 998 & 924 \\
$\mu(\mathrm{mPa} \cdot \mathrm{s})$ & 0.89 & 1.33 & 2.05 & 3.0 & 0.93 & 4.25 \\
$\sigma(\mathrm{mN} / \mathrm{m})$ & 23.4 & 24.9 & 26.0 & 27.0 & 72.0 & 18.6 \\
$D(\mu \mathrm{m})$ & 300 & 300 & 300 & 300 & $300 / 600$ & $300 / 450$ \\
$O h$ & 0.0127 & 0.0178 & 0.0292 & 0.0435 & $0.0069 /$ & $0.0483 /$ \\
& & & & & 0.0044 & 0.0592 \\
\hline
\end{tabular}

Table 2 - Physical properties of S386 solution with various concentrations.

\begin{tabular}{c|ccccc}
\hline S386 (wt. \%) & $0.005 \%$ & $0.25 \%$ & $0.4 \%$ & $1.0 \%$ & $2.0 \%$ \\
\hline$\rho\left(\mathrm{kg} / \mathrm{m}^{3}\right)$ & 996 & 996 & 996 & 997 & 998 \\
$\mu(\mathrm{mPa} \cdot \mathrm{s})$ & 0.92 & 0.95 & 0.98 & 1.01 & 1.09 \\
$\sigma(\mathrm{mN} / \mathrm{m})$ & 32.0 & 22.5 & 21.6 & 21.3 & 21.3 \\
$D(\mu \mathrm{m})$ & 450 & 450 & 450 & 450 & 450 \\
$O h$ & 0.0077 & 0.0095 & 0.0099 & 0.0103 & 0.0111 \\
\hline
\end{tabular}

Transition boundary from regime III to V: the re-derived model for surfactant-free cases The expressions (1), (2) and (3) indicate that $B$ on the transition boundary (III/V) decreases with increasing $W e$ and becomes larger with increased viscosity. The trends of these results are consistent with the experimental data in Refs. 5, 7 and 8, indicating that larger impact inertia encourages stretching separation whereas higher viscosity suppresses it. However, since the coefficients of $\frac{\mu}{\sigma}\left(\frac{\rho D}{\sigma}\right)^{\frac{1}{2}}$ in (1) and (2) have units, $\mathrm{m}^{2} / \mathrm{s}^{2}$, we revisit the derivation and 
see if any dimensionless coefficients can be obtained and lead to a more general form. According to (15) in Ref. 9, the momentum theory for sliding plates was proposed as:

$$
\frac{4}{3} \pi \rho r^{3} U_{n}=\int_{0}^{\tau}(F+D r) d t
$$

where $U_{n}=U B, F \sim(\sigma / b)(a b), D r \sim \mu U_{n} r$, and $\tau \sim r / U_{n}$. By substituting $U_{n}, F, D$ and $\tau$ into (3), and taking $U=\sqrt{\frac{\sigma W e}{\rho d}}$, the equation (4) can be obtained as follows:

$$
\frac{2}{3} \pi W e B^{2}=\left(C_{1} \frac{a}{r}+C_{2} o h \sqrt{W e} B\right)
$$

Here $C_{1}$ and $C_{2}$ are constants, and $\sqrt{W e} B$ can be solved by the quadratic formula as

$$
\begin{aligned}
& \sqrt{W e} B=\frac{C_{2}^{\prime} O h+\sqrt{\left(C_{2}^{\prime} O h\right)^{2}+4 C_{1}^{\prime}}}{2}, \\
& \text { where } C_{1}^{\prime}=\frac{3 C_{1} a}{2 \pi r}, C_{2}^{\prime}=\frac{3 C_{2}}{2 \pi} \text {. Then we get } \\
& B=\frac{1}{2 \sqrt{W e}}\left[C_{2}^{\prime} O h+\sqrt{\left(C_{2}^{\prime} O h\right)^{2}+4 C_{1}^{\prime}}\right] .
\end{aligned}
$$

The basic form of (7) is the same as that proposed by Jiang et al., Gotaas et al. and BrazierSmith et al., showing $B \propto W e^{-0.5}$ (or $W e^{0.5} \propto B^{-1}$ ). However, both $C_{1}^{\prime}$ and $C_{2}^{\prime}$ in (7) are dimensionless, whereas the coefficient used in the model of Jiang et al. and Gotaas et al. are dimensional. The validity of (7) versus various fluid properties is discussed in the later section.

\section{Results and Discussion}

To justify the accuracy of the present models of transition boundary III/V for different fluid properties, (2), (3) and (7) are plotted in Figs. 2 and 3 and compared to the experimental data of water, alkanes and silicone oil drops. Here $C_{1}^{\prime}=8.0$ and $C_{2}^{\prime}=15.0$ in (7), which are obtained by least squares regression. Compared to the models of Gotaas et al. [13] and Finotello et al. [19], the present model with the fitting coefficients results in a good agreement with the experimental data as shown in Figs. 2 and 3. Moreover, for liquids giving relatively high $\mathrm{Oh}$ such as silicone oil drops with different diameters, as shown in Figs. 3(e) and 3(f), (7) gives a good agreement of the transition boundary III/V with the experimental data. However, compared to the coefficients 2.656 and 0.185 in (2), $C_{1}^{\prime}$ and $C_{2}^{\prime}$ in the present model are dimensionless, and these appear to be more reasonable in a dimensionless model.

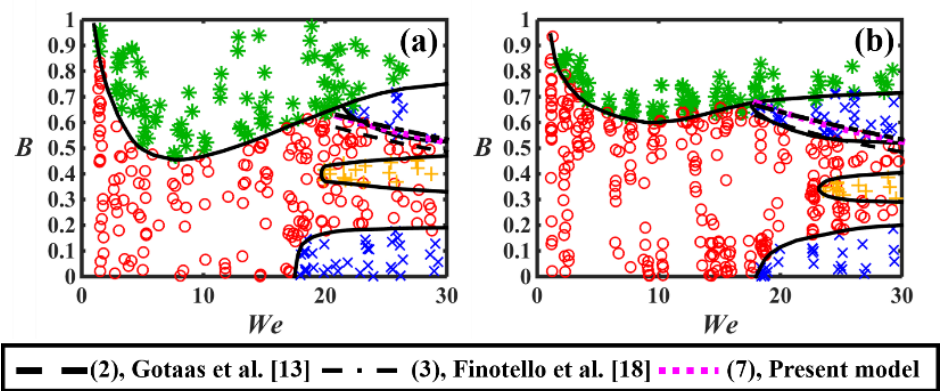

Figure 2. Regime diagrams for water drops with (a) $D=600$ um $(O h=0.0044)$ and (b) 300 um $(O h=0.0069)$. The symbols $\bigcirc$ : coalescence, $X$ : stretching/reflexive separation, *: bouncing, + : rotational separation. 

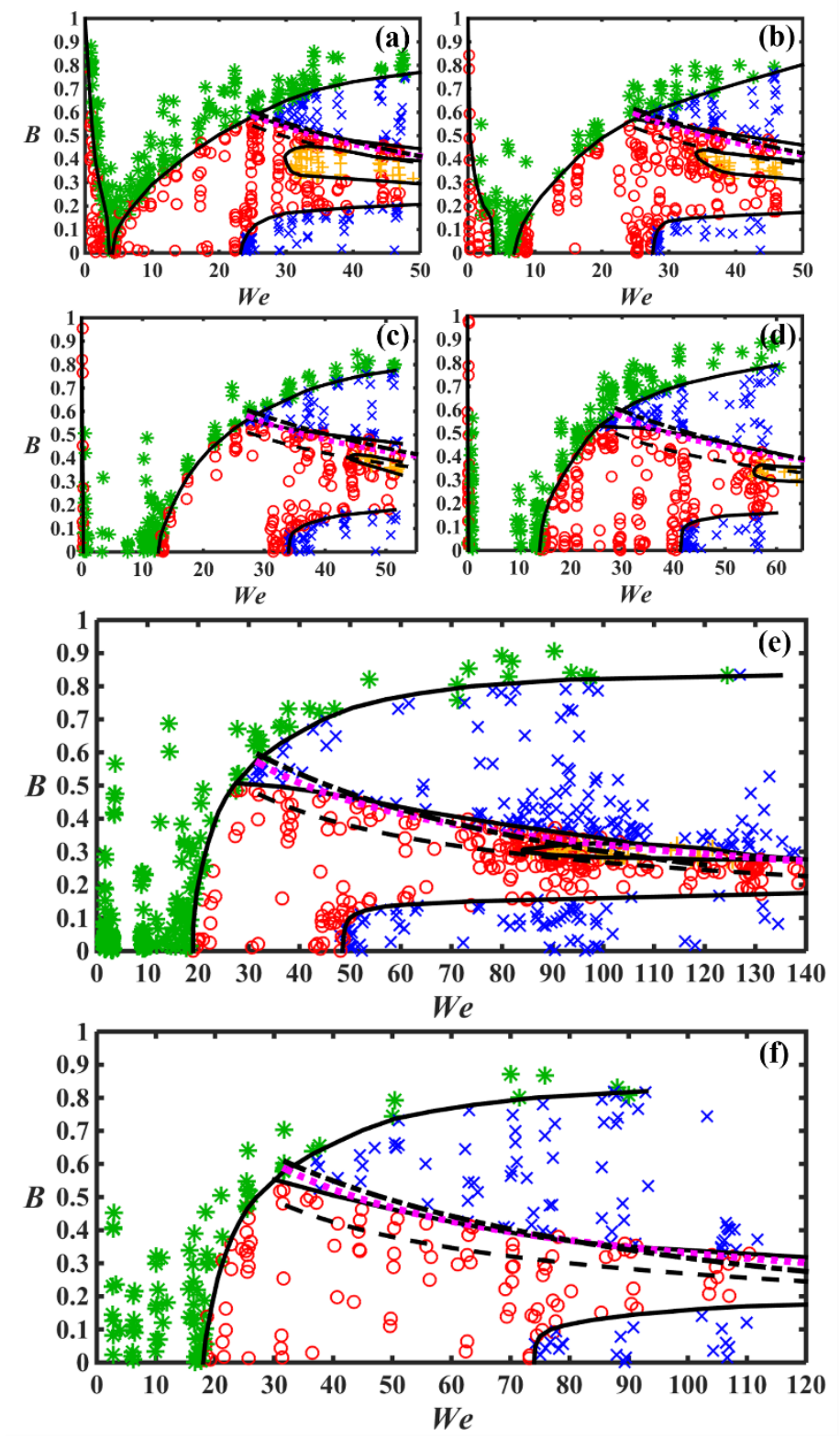

- (2), Gotaas et al. [13] - - (3), Finotello et al. [18] * * (7), Present model

Figure 3. Regime diagrams for (a) decane drops with $D=300$ um and $O h=0.0127$, (b) dodecane drops with $D=$ 300 um and $O h=0.0178$, (c) tetradecane drops with $D=300$ um and $O h=0.0292$, (d) hexadecane drops with $D$ $=300 \mathrm{um}$ and $O h=0.0435$, (e) silicone olil drops with $D=300 \mathrm{um}$ and $O h=0.0483$, and (f) hexadecane drops with $D=300$ um and $O h=0.0592$.

Regarding the influence of surfactant on droplet breakup, by comparing Fig. 4(a) and Fig. 2(a), it shows that a similar $O h$ value, adding a small amount of surfactant $(0.005$ wt.\%) can substantially enlarge the $W e-B$ range of the coalescence regime (Fig. $4 \mathrm{a})$. The experimental data in Fig. 5 further shows that the transition Weber number $\left(W e_{c}\right)$ between regime (III) and (IV) is about 2.2 times larger than the surfactant-free case. When surfactant concentration increases to 0.25 wt.\%, as shown in Fig. 2(b), We further increases, significantly delaying the occurrence of the reflexive separation (IV). Fig. 5 shows that $W e_{c}$ reaches the maximum value at $\Phi=0.25$ wt. $\%$, which is about 2.6 times larger than that of the surfactant-free case. To note that, when $\Phi>0.25 \mathrm{wt} . \%$, the bouncing regime was enlarged due to the Marangoni effect prohibiting air drainage between two approaching droplets [21]. 
By increasing $\Phi$ to $0.4 \mathrm{wt} . \%$, however, the suppression on regime (IV) is retarded, as shown in Fig. 4(c). It shows that $W e_{c}$ no longer increases but decreases. When $\Phi$ further increases to 1.0 and 2.0 wt.\%, as shown in Fig. 4(d) and Fig 4(e), respectively, We keeps decreasing as $\Phi$ increases. Fig. 5 further shows that by increasing S386 concentration when $\Phi>0.25$ $w t . \%, W e_{c}$ gradually approaches the prediction of the previous model [10]. For the off-center collision, at $\Phi=0.005$ wt. $\%$, Fig 4(a) shows that the $B$ values at the transition boundary between regime (III) and (V) are $45 \%$ larger than the theoretical predictions of (7) at a fixed We. When $\Phi \geq 0.25$ wt.\%, as shown in Figs. 4(b) - 4(e), the experimental data are $30 \%$ larger than the theoretical values. These results show that the addition of surfactant can also suppress the stretching separation.

Previous studies of droplet breakup in a shear flow [23, 24] have shown that, with the surfactant concentration gradients on the droplet interface, the Marangoni stresses retarded the breakup, while this mechanism could further elucidate our experimental finding. After droplet impact, the reflective or stretching flow motion elongates the tentatively merged drop, wiping out the surfactant molecules to the end of the drop and generate the Marangoni stresses toward the drop center. These additional stresses pull back the elongated drop and consequentially retard droplet breakup. Thus, it is necessary to further include the Marangoni force in the theoretical models to predict the transition boundaries between coalescence and various separation regimes under the influence of surfactant.
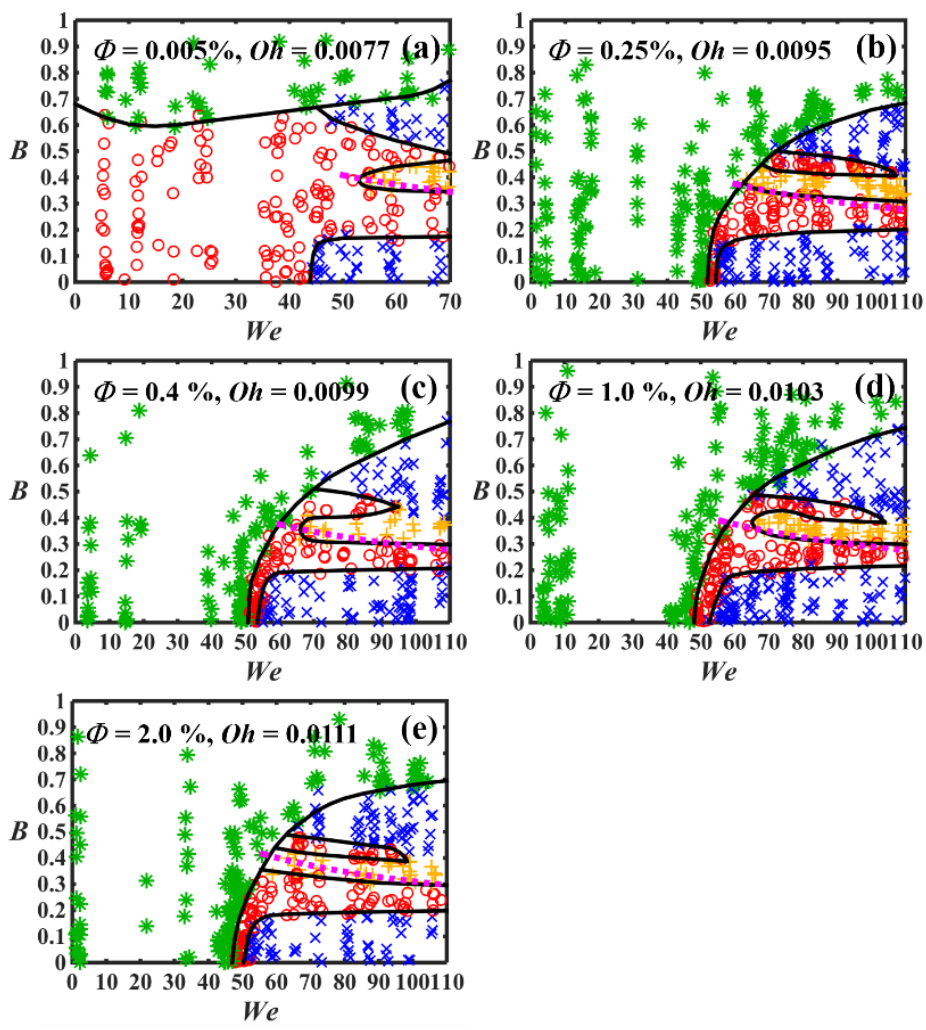

Figure 4. Regime diagrams for drops ( $D=450 \mathrm{um}$ ) with various concentration of S386. (a) 0.005 wt. $\%$ and $\mathrm{Oh}=$ 0.0077 , (b) 0.25 wt. $\%$ and $O h=0.0095$, (c) 0.4 wt. $\%$ and and $O h=0.0099$, (d) 1.0 wt. $\%$ and $O h=0.0103$, (e) 2.0 wt. $\%$ and $O h=0.0111$ 


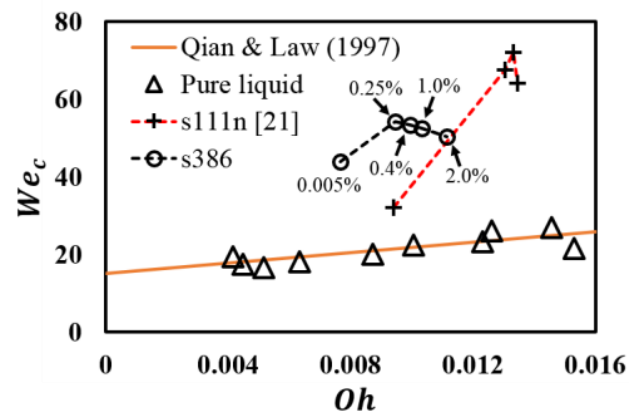

Figure 5. Experimental measurements of $W e_{c}$ compared to the model derived by Qian and Law for $W e_{c}$.

\section{Conclusions}

The sliding plate model proposed by Jiang et al. [9] to predict the transition boundary from coalescence to stretching separation in binary droplet collision has been re-derived as the following form:

$$
B=\frac{1}{2 \sqrt{W e}}\left[C_{2}^{\prime} O h+\sqrt{\left(C_{2}^{\prime} O h\right)^{2}+4 C_{1}^{\prime}}\right]
$$

where $C_{1}^{\prime}=8.0$ and $C_{2}^{\prime}=15.0$. The results show an excellant agreement with the experimental data of surfactant-free drops in a wide range of $O h$ from 0.0089 to 0.0592 . Furthermore, in contrast to the coefficients in the previous models $[9,13]$ that have units $\mathrm{m}^{2} / \mathrm{s}^{2}$, the coefficients $C_{1}^{\prime}$ and $C_{2}^{\prime}$ are dimensionless and are consistent with a model generically expressed by dimensionless parameters. On the other hand, we have demonstrated that adding surfactant delays the occurrence of reflexive separation (IV), where the maximum $W e_{c}$ can be 2.6 times larger than the theoretical prediction of a surfactant-free case. Regarding off-center collisions, the surfactant cases need at least $30 \%$ larger $B$ to reach stretching separation than the cases without surfactant. The suppression of the separations by adding surfactant in droplet collisions could result from additional stresses exerted by the non-uniform distribution of surfactant on the droplet surface [23, 24], while the details need to be confirmed by further studies.

\section{Acknowledgments}

We thank the funding support of the Ministry of Science and Technology, Taiwan (MOST 1092221-E-002-203).

\section{Nomenclature}

$\begin{array}{ll}B & \text { impact parameter (dimensionless) } \\ D & \text { droplet diameter [mm] } \\ D r & \text { viscous force }[\mathrm{N}] \\ F & \text { surface tension force }[\mathrm{N}] \\ O h & \text { Ohnesorge number } \\ U & \text { relative velocity }\left[\mathrm{m} \mathrm{s}^{-1}\right] \\ W e & \text { Weber number } \\ \rho & \text { density }\left[\mathrm{kg} \mathrm{m}^{-3}\right] \\ \mu & \text { liquid viscosity }[\mathrm{mPa} \mathrm{s}] \\ \Phi & \text { surfactant concentration } \\ \sigma & \text { surface tension }\left[\mathrm{mN} \mathrm{m}^{-1}\right]\end{array}$

\section{References}


[1] Gunn, R., 1965, Science, 150, pp. 695.

[2] Strangeways, I., 2006, "Precipitation: theory, measurement and distribution."

[3] Chiu, H. H., 2000, Progress in Energy Combustion Science, 26, pp. 381.

[4] Zhang, D., Zhang, P., Yuan, Y., and Zhang, T., 2016, Combustion and Flame, 173, pp. 276.

[5] Moretto, L. G., Tso, K. Colonna, N., and Wozniak, G. J., 1992, Physical Review Letters, 69, pp. 1884.

[6] Bauer, W., Bertsch, G. F., and Schulz, H., 1992, Physical Review Letters, 69, pp. 1888.

[7] Brazier-Smith, P. R., Jennings, S. G., and Latham, J., 1972, Proceedings of the Royal Society of London A, 326, pp. 393-408.

[8] Ashgriz, N., and Poo, J. Y., 1990, Journal of Fluid Mechanics, 221, pp. 183-204.

[9] Jiang, Y. J., Umemura, A., and Law, C. K., 1992, Journal of Fluid Mechanics, 234, pp. 171190.

[10] Qian, J., and Law, C. K., 1997, Journal of Fluid Mechanics, 331, pp. 59-80.

[11] Pan, K. L., Chou, P. C., and Tseng, Y. J., 2009, Physical Review E, 80, 036301.

[12] Pan, K. L., Huang, K. L., Hsieh, W. T., and Lu, C. R., 2019, Physical Review Fluids, 4, pp. 12306.

[13] Gotaas, C., Havelka, P., Jakobsen, H. A., Svendsen, H. F., Hase, M., Roth, N., and Weigand, B., 2007, Physics of Fluid, 19, 102106.

[14] Huang, K. L., Pan, K. L., and Josserand, C., 2019, Physical Review Letters, 123, pp. 234502.

[15] Rabe, C., Malet, J., and Feuillebois, F., 2010, Physics of Fluids (1994-present), 22(4), pp. 047101.

[16] Estrade, J. P., Carentz, H., Lavergne, G., and Biscos, Y., 1999, International Journal of Heat and Fluid Flow, 20(5), pp. 486-491.

[17] Kuschel, M., and Sommerfeld, M., 2013, Experiments in Fluids, 54, pp. 1440.

[18] Sommerfeld, M., and Kuschel, M., 2016, Experiments in Fluids, 57, pp. 181.

[19] Finotello, G., Padding, J. D., Deen, N. G., Jongsma, A., Innings, F., and Kuipers, J. A. M., 2017, Physics of Fluids, 26, pp. 067102.

[20] Finotello, G., Kooiman, R. F., Padding, J. T., Buist, K. M., Jongsma, A., Innings, F., and Kuipers, J. A. M., 2018, Experiments in Fluids, 59, pp. 17.

[21] Pan, K. L., Tseng, Y. H., Chen, J. C., Huang, K. L., Wang, C. H., and Lai, M. C., 2016, Journal of Fluid Mechanics, 799, pp. 603-636.

[22] Al-Dirawi, K. H., and Bayly, A. E., 2019, Physics of Fluids, 31, pp. 027105.

[23] Stone, H. A., and Leal, L. G., 1990, Journal of Fluid Mechanics, 220(161), pp.161-186.

[24] Drumright-Clarke, M. A., and Renardy, Y., 2004, Physics of fluids, 16(1), pp. 14-21. 